\title{
Unilateral Nonvisualization of a Transverse Dural Sinus on Phase-Contrast MRV: Frequency and Differentiation from Sinus Thrombosis on Noncontrast MRI
}

\author{
(D).-M. Chang, (D) A.L. Kuhn, (D) N. Porbandarwala, (D) R. Rojas, (D) V. Ivanovic, and (DR.A. Bhadelia
}

\begin{abstract}
BACKGROUND AND PURPOSE: Unilateral decreased/nonvisualization of a transverse dural sinus on MRV poses a diagnostic dilemma when gadolinium administration is contraindicated. We determined the frequency of unilateral decreased/nonvisualization of the transverse dural sinus and the performance of pregadolinium MR imaging sequences in diagnosing transverse sinus thrombosis in the presence of unilateral decreased/nonvisualization on phase-contrast MRV.
\end{abstract}

MATERIALS AND METHODS: We conducted a retrospective review of consecutive 3D phase-contrast MRV (VENC, $30 \mathrm{~cm} / \mathrm{s})$ and routine brain imaging (noncontrast sagittal T1, axial T2, FLAIR, DWI, GRE, and postgadolinium 3D-MPRAGE images) performed during a 3-year period for a total of 208 patients. Nonvisualization of a transverse dural sinus was defined as $\geq 50 \%$ nonvisualization of the transverse sinus caliber versus the contralateral side on MRV. Noncontrast imaging findings were considered abnormal when hyperintense signal was present on T2, FLAIR, T1, and DWI, and there were T2* blooming artifacts on GRE and DWI. Postgadolinium 3D-MPRAGE was used to confirm the diagnosis of transverse sinus thrombosis.

RESULTS: Nonvisualization of a transverse dural sinus was observed in 72/208 (34.6\%) patients on MRV; 56/72 (77.8\%) were without transverse sinus thrombosis, and 16/72 (22.2\%) patients had transverse dural sinus thrombosis. Nonvisualization of a transverse dural sinus was seen in 56/192 (29.2\%) patients without transverse sinus thrombosis and 16/16 (100\%) with transverse sinus thrombosis. Abnormal findings on DWI (transverse sinus hyperintense signal or T2* blooming artifact) are $93.8 \%$ sensitive and $100.0 \%$ specific for transverse sinus thrombosis. Other noncontrast MR imaging sequences ranged from $56.3 \%-68.8 \%$ sensitive and $91.1 \%-100.0 \%$ specific.

CONCLUSIONS: Nonvisualization of a transverse dural sinus is a frequent phenomenon on phase-contrast MRV. DWI can be effectively used to exclude sinus thrombosis when nonvisualization of a transverse dural sinus is a diagnostic conundrum on phase-contrast MRV and contrast-enhanced studies are contraindicated.

ABBREVIATIONS: $\mathrm{CE}=$ contrast-enhanced; $\mathrm{CVT}=$ cerebral venous thrombosis; DST $=$ dural venous sinus thrombosis; MPRAGE $+=$ postgadolinium $3 \mathrm{D}-$ MPRAGE; MRI- = noncontrast MRI brain sequences; NV = unilateral decreased/nonvisualization; NVTS $=$ nonvisualization of a transverse dural sinus; $\mathrm{PC}=$ phase-contrast; TS $=$ transverse dural sinus; TST $+=$ transverse sinus thrombosis; TST $-=$ no transverse sinus thrombosis

D ural venous sinus thrombosis (DST) and cerebral venous thrombosis (CVT) are reportedly uncommon, with an annual incidence of 3-4 per 1 million to 1.32 per 100,000 and represent approximately $0.5 \%-1.0 \%$ of all strokes. ${ }^{1-3}$ Young and middleaged adults are disproportionately affected; within this age group, DST and CVT are 3 times more common in women. ${ }^{4}$ While the etiologies include infection, trauma, iatrogenic causes, and genetic

Received June 20, 2019; accepted after revision October 11

From the Division of Neuroradiology, Department of Radiology, Beth Israel Deaconess Medical Center, Boston, Massachusetts.

Please address correspondence to Yu-Ming Chang, MD, PhD, Department of Radiology, Beth Israel Deaconess Medical Center, WCB90, 330 Brookline Ave, Boston, MA 02115; e-mail: ychang2@bidmc.harvard.edu

http://dx.doi.org/10.3174/ajnr.A6337 predisposition to hypercoagulability, this prominent sex predisposition is thought to be secondary to oral contraceptive use and, most important, pregnancy and peripartum states. ${ }^{4}$

Although rare, delayed diagnosis of DST and CVT could result in devastating clinical consequences, including venous infarct and intracranial hemorrhages, leading to high morbidity and mortality rates between $5 \%$ and $10 \% .{ }^{3,5}$ There is a highly variable clinical presentation, ranging from asymptomatic, headache, focal neurologic deficits, intracranial hypertension, and seizures. ${ }^{4}$ Of DST and CVT, DST is more frequent, and of these, transverse dural sinus (TS) thrombosis is among the most common, with $44.8 \%$ involvement of the left TS and $41.3 \%$ of the right TS per 1 report and up to $70 \%$ (side not specified) in another series. ${ }^{2,6}$

Given the variable and nonspecific clinical presentation of DST and the importance of rapid treatment, imaging is essential 


\begin{tabular}{lcccc}
\hline & \multicolumn{3}{c}{ No Thrombosis } & \\
\cline { 2 - 3 } & \multicolumn{2}{c}{ Normal TS } & NVTS & Thrombosis \\
\hline Total (No.) & 136 & 56 & 16 \\
Mean age (range) $(y r)$ & $42.9(16-83)$ & $48.6(27-90)$ & $42.3(21-87)$ \\
Patients (No.) & $136(79.4 \%$ Female) & $56(58.9 \%$ Female $)$ & $16(68.8 \%$ Female) \\
\hline
\end{tabular}

of each other was conducted. Exclusion criteria included known preexisting DST and CVT and a recent operation involving the cranium. At our institution, initial MR imaging assessment for suspected DST was made with PCMRV because CE-MRV had not been implemented during the study period.

in its diagnosis. ${ }^{7}$ Contrast-enhanced MR imaging techniques, such as contrast-enhanced MRV (CE-MRV), followed by 3D T1weighted imaging and CVT are now considered the criterion standard for imaging diagnosis of DST, demonstrating superiority over noncontrast MRV, MR imaging, and CT..$^{7-12}$

However, the use of gadolinium contrast may be contraindicated or relatively contraindicated in patients with renal failure and in pregnant patients, given the concerns for nephrogenic systemic fibrosis and tetratogenic effects on the fetus, respectively. Most important, this concern limits the use of gadolinium in pregnant patients, a group with increased risk for the development of DST. CVT may also be refused by pregnant patients due concerns secondary to the use of ionizing radiation, despite negligible fetal exposure for head examinations. ${ }^{13}$ Furthermore, there has been increasing concern among the public regarding gadolinium deposition of entirely uncertain clinical significance in patients with normal renal function, particularly in deep brain structures. ${ }^{14}$ This has led many patients to refuse gadolinium out of concern for bodily harm.

In particular, diagnosis and subsequent appropriate treatment for transverse sinus thrombosis (TST + ) can be challenging with noncontrast MRV. Well-established pitfalls includes false-positive diagnoses due to difficulty in distinguishing nonvisualization of a transverse dural sinus (NVTS) secondary to congenital hypoplasia, slow flow due to downstream compression of the brachiocephalic or internal jugular veins, direction of venous flow, as well as idiopathic intracranial hypertension from true thrombosis. ${ }^{15-19}$ While standard noncontrast MR imaging head sequences can be insensitive compared with CE-MRV and CVT for detection of DST, ${ }^{12}$ we hypothesize that standard MR imaging head sequences may be highly sensitive and specific in differentiating thrombosis from other causes of NVTS when applied to equivocal noncontrast MRVs, significantly increasing the diagnostic confidence in clinical scenarios where contrast or ionizing radiation are contraindicated or refused. Our purpose was to determine the frequency of unilateral NVTS and the performance of pregadolinium MR imaging sequences in diagnosing TST + in the setting of unilateral decreased/nonvisualization (NV) on phase-contrast MRV (PC-MRV).

\section{MATERIALS AND METHODS \\ Patient Selection}

Following institutional review board approval for this Health Insurance Portability and Accountability Act-compliant study, a retrospective review from 2014 to 2017 of our institutional imaging data base for noncontrast 3D-PC-MRV (VENC, $30 \mathrm{~cm} / \mathrm{s}$ ) with additional standard MR imaging brain sequences (spin-echo sagittal T1, axial T2, axial GRE, axial FLAIR, axial DWI, and postgradolinium 3D-MPRAGE [MPRAGE+]) performed within 24 hours
Standard MR imaging brain sequences (including MPRAGE + ) were subsequently performed on patients whose clinical presentations required additional imaging to assess intracranial abnormalities including, but not limited to, infarct, hemorrhage, mass, infection/inflammation, or high suspicion for DST. Two hundred eight patients fitting the inclusion criteria were identified. MPRAGE + served as the diagnostic criterion standard for TST+ versus NV of PC-MRV signal. Demographic characteristics of the patients are shown in Table 1.

\section{Imaging Protocols}

All MR imaging examinations were performed on either a $1.5 \mathrm{~T}$ Signa HDx scanner (GE Healthcare, Milwaukee, Wisconsin) or a 1.5T Magnetom Espree scanner (Siemens, Erlangen, Germany) using standard 8- and 12-channel head coils, respectively.

The Signa HDx scanner imaging parameters (TR/TE/flip angle/section thickness/matrix) were the following: sagittal T1 $\left(650 / 13 \mathrm{~ms} / 90^{\circ} / 5 \mathrm{~mm} / 256 \times 192\right)$, axial T2 $\left(4200 / 11 \mathrm{~ms} / 90^{\circ} /\right.$ $5 \mathrm{~mm} / 320 \times 224)$, axial GRE $\left(550 / 9.1 \mathrm{~ms} / 20^{\circ} / 5 \mathrm{~mm} / 320 \times\right.$ 224), axial FLAIR $\left(8600 / 80 \mathrm{~ms} / 150^{\circ} / 5 \mathrm{~mm} / 320 \times 192\right)$, axial DWI $\left(8000 / 96 \mathrm{~ms} / 90^{\circ} / 5 \mathrm{~mm} / 128 \times 128\right), \mathrm{MPRAGE}+(7.7 /$ $\left.3.3 \mathrm{~ms} / 15^{\circ} / 1 \mathrm{~mm} / 240 \times 224\right)$, and 3D PC-MRV $(9.83 / 4.3 \mathrm{~ms} /$ $\left.90^{\circ} / 1.8 \mathrm{~mm} / 256 \times 224\right)$.

Imaging parameters (TR/TE/flip angle/section thickness/matrix) for the Magnetom Espree scanner included the following: sagittal T1 $\left(420 / 8.8 \mathrm{~ms} / 90^{\circ} / 5 \mathrm{~mm} / 256 \times 100\right)$, axial T2 $(4500 /$ $\left.95 \mathrm{~ms} / 150^{\circ} / 5 \mathrm{~mm} / 384 \times 88\right)$, axial GRE $\left(835 / 26 \mathrm{~ms} / 20^{\circ} / 5 \mathrm{~mm} /\right.$ $256 \times 75)$, axial FLAIR $\left(9000 / 84 \mathrm{~ms} / 170^{\circ} / 5 \mathrm{~mm} / 256 \times 100\right)$, axial DWI $\left(4600 / 103 \mathrm{~ms} / 90^{\circ} / 5 \mathrm{~mm} / 130 \times 100\right)$, MPRAGE + $\left(2100 / 3.57 \mathrm{~ms} / 15^{\circ} / 1 \mathrm{~mm} / 256 \times 100\right)$, and 3D PC-MRV (43.5/ $\left.6.86 \mathrm{~ms} / 15^{\circ} / 1.8 \mathrm{~mm} / 256 \times 88\right)$.

We administered $0.1 \mathrm{mmol} / \mathrm{kg}$ of gadobutrol (Gadavist; Bayer Schering Pharma, Berlin, Germany), with a maximum of $10 \mathrm{~mL}$ at a rate of $1.5 \mathrm{~mL} / \mathrm{s}$ for postcontrast imaging.

\section{Image Analysis}

Two hundred eight PC-MRVs were reviewed for the presence of NVTS by 2 neuroradiologists separately, blinded to the final reported diagnosis and without access to MPRAGE + or standard MR imaging brain sequences: A.L.K. (neuroradiology fellow) and R.A.B. (28 years of experience), with no disagreements. Before data analysis, A.L.K. was trained by R.A.B. using a series of 5 representative PC-MRVs with NVTS and 5 PC-MRVs with normal findings (as defined by R.A.B.) in association with corresponding MPRAGE images that were not obtained within the study date range. These representative examples were then used as a reference during the analysis period. The TS was categorized as normal or NV, with laterality recorded. TS was defined as NV on PC-MRV when the lack of flow-related signal was $\geq 50 \%$ 

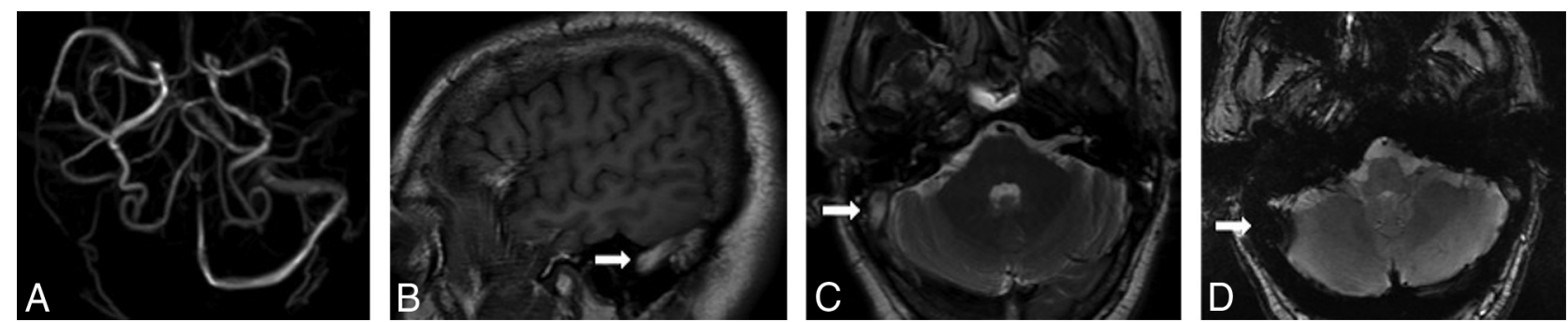

FIG 1. Absence of the right transverse and sigmoid sinuses on PC-MRV $(A)$. Abnormal hyperintense signal in the right transverse and sigmoid sinuses on sagittal $\mathrm{Tl}(B$, arrow) and axial T2 (C, arrow), with corresponding T2*-hypointense signal with blooming artifacts on axial GRE sequences (D, arrow).
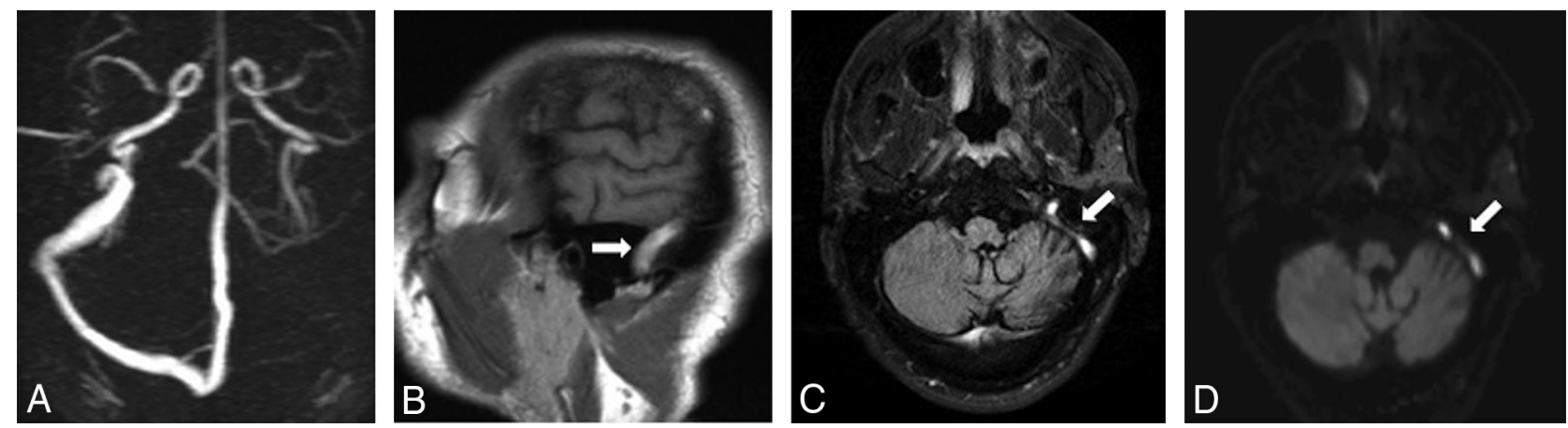

FIG 2. Absence of the left transverse and sigmoid sinuses on PC-MRV (A). Abnormal hyperintense signal in the left transverse and sigmoid sinuses on sagittal Tl ( $B$, arrow) and axial FLAIR sequences ( $C$, arrow) with corresponding high signal on DWI (D, arrow).

relative to contralateral TS. ${ }^{16}$ Nonocclusive short-segment and rounded filling defects in the TS were not considered abnormal because these findings are typically attributable to arachnoid granulations. ${ }^{18,20}$

The presence or absence of TST + was then confirmed in both groups with corresponding MPRAGE+ sequences by R.A.B. and A.L.K., separately, with no disagreements. A normal TS on PCMRV was confirmed to be free of thrombosis.

For all patients with NVTS on PC-MRVs, the signal characteristics of the bilateral TS on pregadolinium images (sagittal T1, axial T2, axial GRE, axial FLAIR, and axial DWI) were further categorized as normal or abnormal by 2 neuroradiologists blinded to the results of PC-MRV analysis, for the presence or absence of TS thrombosis without access to MPRAGE+ images (R.R., with 20 years of experience and V.I. with 12 years of experience). R.R. and V.I. did not receive additional training before the analysis regarding thresholds for considering abnormal TS signal. Evaluation of abnormal TS signal on standard noncontrast MR imaging brain sequences (MRI-) was performed on only patients with NVTS on PC-MRV because those with a normal TS on PCMRV had negative findings for TST + .

Pregadolinium imaging findings were considered normal if the TS signal was iso- to hypointense on T1, T2, and FLAIR and hyperintense on GRE to the cortex. TS signal isointense to CSF was considered a normal finding on DWI. Pregadolinium imaging findings were considered abnormal when hyperintense signal was present on T1, T2, FLAIR, and DWI and T2*-hypointense signal with blooming artifacts (T2* blooming artifacts) was present with expanded TS on GRE and DWI (Figs 1-4). NVTS on
PC-MRV was correlated to the anatomic size of the TS at the level of the sigmoid notch relative to the contralateral side on MPRAGE + . TS size measurements were performed by 1 neuroradiologist (Y.-M.C.) with 4 years of experience.

\section{Data Analysis}

Interobserver reliability for the presence of NVTS, TST + on MPRAGE + , and normal-versus-abnormal TS signal on noncontrast MRI brain sequences (MRI-) in patients with NVTS on PC-MRV was assessed with $\kappa$ coefficients. The side of abnormal TS signal on MR imaging was recorded. Disagreements were resolved by consensus review, and the reconciled data were used for subsequent analysis.

The association between sizes of the TS and TST + was calculated with a $\chi^{2}$ test.

The frequency and laterality of abnormal MRI- TS signal were determined for NVTS without transverse sinus thrombosis (TST-) and NVTS with TST + . The sensitivity and specificity of abnormal MRI- TS signal for individual sequences in patients with NVTS and for all sequences combined excluding DWI (ie, if any sequence had abnormal findings) in confirming TST + in the setting of NVTS on PC-MRV, were determined. Statistical significance was considered with $P \leq .05$.

\section{RESULTS}

By means of terminology established by Landis and Koch, ${ }^{21}$ there was perfect agreement between readers in detecting NVTS on PC-MRV and the presence of TST + on MPRAGE $+(\kappa=1.0$, respectively). There was substantial agreement in detecting GRE 

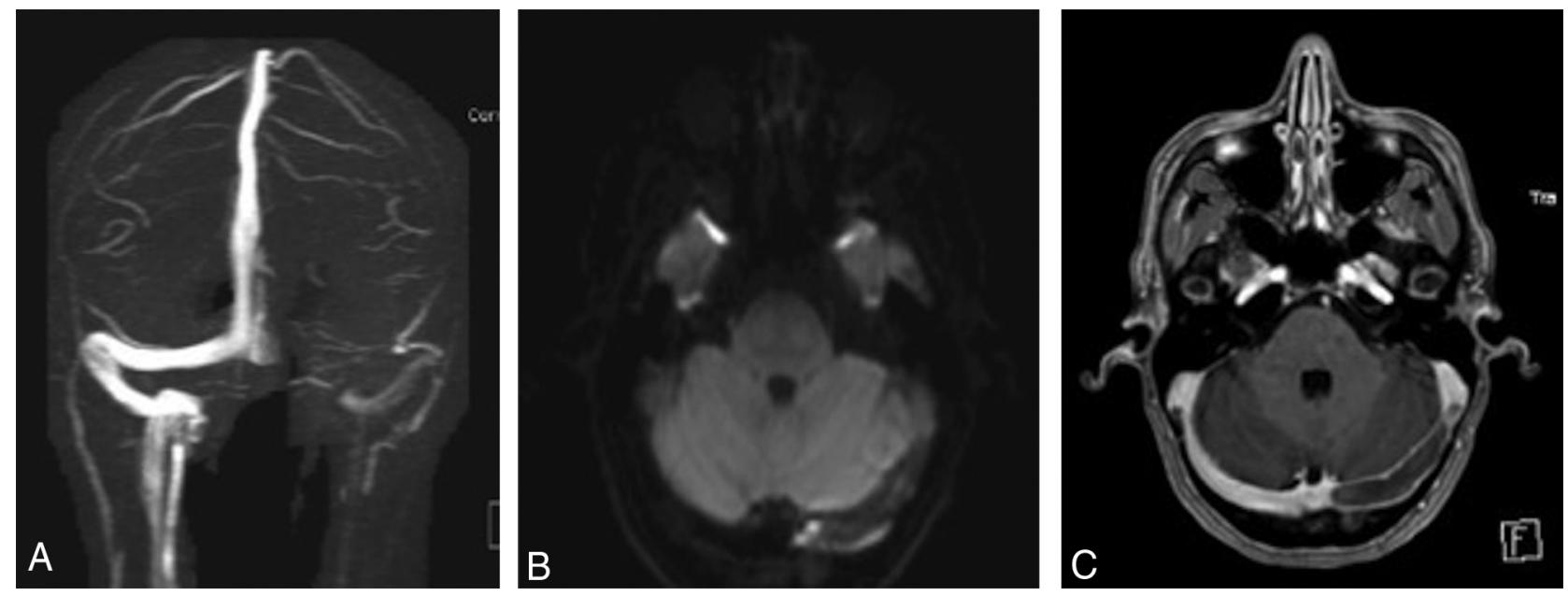

FIG 3. Representative images of a patient demonstrating $T 2^{*}$-hypointense signal with blooming artifacts on DWI. Absence of the left transverse sinus on PC-MRV $(A)$ with associated T2*-hypointense signal with blooming artifacts on DWI (B), corresponding to a filling defect on axial MPRAGE (C).
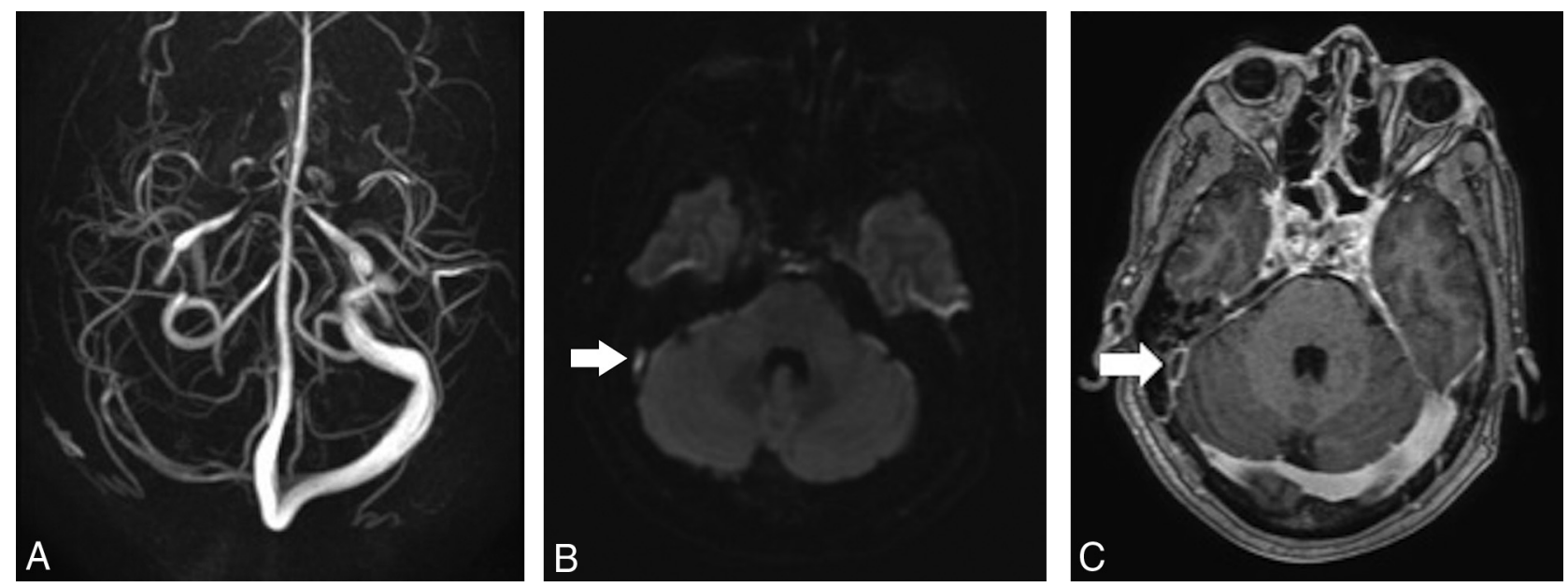

FIG 4. Representative images of a patient different from the one in Fig 3, demonstrating DWI hyperintense signal. Absence of the right transverse and sigmoid sinuses on PC-MRV $(A)$, with associated hyperintense signal on DWI (B, arrow), corresponding to a filling defect on axial MPRAGE (C, arrow).

signal abnormality $(\kappa=0.78)$ and almost perfect agreement in detecting T1 $(\kappa=0.85)$, T2 $(\kappa=0.95)$, FLAIR $(\kappa=0.97)$, and DWI $(\kappa=0.86)$ signal abnormalities.

NVTS on PC-MRV was observed in 72/208 (34.6\%) patients, and 16 of these 72 patients demonstrated TST + on MPRAGE + . NVTS was seen in 56/192 (29.2\%) patients with TST- and 16/16 (100\%) with TST+.

In NVTS without transverse sinus thrombosis, 11 right TSs and 45 left TSs were not visualized. Relative size comparison of the TS at the level of the sigmoid notch on MPRAGE showed that the right TS was smaller than the left in 6 cases, the left TS was smaller than the right in 46 cases, with the right and left TSs were equal in 4 cases. Although 51/56 (91.1\%) NVTSs with TSTwere associated with a smaller size of the TS on MPRAGE+, 5/56 $(8.9 \%)$ were not, suggesting other etiologies such as venous slow flow due to downstream effects.

In NVTS with transverse sinus thrombosis, 10 right TSs and 6 left TSs were not visualized secondary to thrombus. Relative size comparison of the TSs showed that the right TS was smaller than the left in 3 cases, the left TS was smaller than the right in 8 cases, with the right and left TS equal in 5 cases. There was no association between the size of the TS and TST $+(P=.42)$.

Signal abnormality on standard MR imaging sequences in cases of NVTS on PC-MRV for TST+ versus TST- as well as sensitivity and specificity for the detection of TST + on T1, T2, FLAIR, GRE, and DWI (hyperintense and T2* blooming artifacts combined) are noted on Table 2. DWI hyperintense signal alone is $43.8 \%$ sensitive $(95 \% \mathrm{CI}, 19.8 \%-70.1 \%)$ and $100.0 \%$ specific (95\% CI, 93.6\%-100.0\%). DWI T2* blooming artifacts alone is $50.0 \%$ sensitive (95\% CI, 24.7\%-75.4\%) and $100.00 \%$ specific (95\% CI, $93.6 \%-100.00 \%)$. A combination of T1, T2, FLAIR, and GRE was $87.5 \%$ sensitive (95\% CI, 61.7-98.5) and $92.9 \%$ specific (95\% CI, 82.7-98.0) for TST+ in NV TS on PC-MRV. The combination of both signal abnormalities on DWI (DWI hyperintense signal and $\mathrm{T}^{*}$ blooming artifacts) is $93.8 \%$ sensitive (95\% CI, $69.8 \%-99.8 \%$ ) and $100.0 \%$ specific (95\% CI, 93.6\%-100.0\%). 
Table 2: TS signal abnormality on standard MR imaging sequences with NVTS on MRV with and without thrombosis

\begin{tabular}{|c|c|c|c|c|c|c|c|c|c|c|}
\hline & \multicolumn{4}{|c|}{ TST + on MPRAGE $+(n=16)$} & \multicolumn{4}{|c|}{ TST- on MPRAGE $+(n=56)$} & \multirow[b]{2}{*}{$\begin{array}{l}\text { Sensitivity } \\
(95 \% \mathrm{CI})(\%)\end{array}$} & \multirow[b]{2}{*}{$\begin{array}{l}\text { Specificity } \\
(95 \% \mathrm{Cl})(\%)\end{array}$} \\
\hline & Low/Iso & Hyper & $\mathrm{T} 2 *$ & $\begin{array}{c}\text { Normal } \\
\text { Signal }\end{array}$ & Low/Iso & Hyper & $\mathrm{T}^{*}$ & $\begin{array}{c}\text { Normal } \\
\text { Signal }\end{array}$ & & \\
\hline T1 sagittal & 7 & 9 & - & - & 56 & 0 & - & - & $56.3(29.9-80.3)$ & $100(93.6-100.0)$ \\
\hline $\mathrm{T} 2 \mathrm{WI}$ & 6 & 10 & - & - & 55 & 1 & - & - & $62.5(35.4-84.8)$ & $98.2(90.5-99.9)$ \\
\hline FLAIR & 5 & 11 & - & - & 51 & 5 & - & - & $68.8(41.3-89.0)$ & $91.1(80.4-97.0)$ \\
\hline GRE & - & 8 & 8 & - & - & 56 & 0 & - & $50.0(24.7-75.4)$ & $100(93.6-100.0)$ \\
\hline DWI & - & 7 & 8 & 1 & - & 0 & 0 & 56 & $93.8^{\mathrm{a}}(69.8-99.8)$ & $100^{a}(93.6-100.0)$ \\
\hline
\end{tabular}

Note:-Iso indicates isointensity; Hyper, hyperintensity; -, no data.

${ }^{a}$ Combined DWI hyperintense and T2* hypointense signal with blooming artifacts.

\section{DISCUSSION}

To the best of our knowledge, for the first time, our results show that DWI signal abnormality, both $\mathrm{T} 2 *$ blooming artifacts and hyperintense signal, can be a powerful diagnostic tool for the clarification of equivocal findings of TST+ on noncontrast MRVs, with a sensitivity of $93.8 \%$ and a specificity of $100 \%$ and interrater reliability of $\kappa=0.86$. While most prior work described much lower sensitivities for the detection of DST with DWI sequences, these studies did not assess $\mathrm{T} 2{ }^{\star}$ blooming artifacts on DWI. ${ }^{12,22}$ However, our results are supported in prior work by Yildiz et $\mathrm{al}^{23}$ on cortical vein thrombosis, which demonstrated sensitivities of up to $84 \%$ on DWI if both hyperintense signal and T2* blooming artifacts were assessed. It is possible that our higher sensitivity for DWI is because the current study aims to clarify a specific diagnostic conundrum in a much larger anatomic structure (TS) on PC-MRV and due to our assessment of both DWI hyperintense signal and $\mathrm{T}^{*}$ blooming artifacts. Thus, in the setting of NVTS on PC-MRV, DWI signal abnormality is virtually diagnostic for TST+, with the interrater reliability ranging from substantial to almost perfect agreement in the acute-to-subacute setting. This may prove to be a powerful tool in overcoming the limitations of commonly used noncontrast MRV (such as 2D-TOF, $3 \mathrm{D}-\mathrm{TOF}$, and PC-MRV) when the use of gadolinium contrast is contraindicated for criterion standard CE-MRV techniques.

The fairly low sensitivity and variable specificity of individual T1, T2, FLAIR, and GRE sequences in the detection of DST has been previously documented, and our results support the earlier findings. For example, prior work in detecting DST in all major dural venous sinuses by Sadigh et $\mathrm{al}^{22}$ found that for 1 reviewer, the sensitivity and specificity, respectively, of T1 noncontrast was $55 \%$ and $55 \%$; for T2, they were $58 \%$ and $97 \%$; for FLAIR, they were $67 \%$ and $62 \%$; for GRE, they were $67 \%$ and $62 \%$; and for DWI (high signal only), they were $25 \%$ and $93 \%$. The above sensitivities are similar to those reported in the current study focusing on the TS: For example, the sensitivity for the detection of TST + is $62.5 \%$ for T2 and $68.8 \%$ for FLAIR. The much higher specificity in our cohort may be secondary to increased pretest probability for TST+, given that our analysis was limited to patients with abnormal findings on PC-MRV. As discussed above, the prominent difference between the current report and previously reported DWI sensitivities is presumed secondary to the inclusion of $\mathrm{T} 2 *$ blooming artifacts on DWI as abnormal in the current study.

A combination of all MRI- sequences (excluding DWI) results in an improved sensitivity of $87.5 \%$ and a decreased specificity of $92.9 \%$, similar to that reported in a large series of
429 patients in which standard MR imaging sequences, including postcontrast sequences, had a combined sensitivity of $79.2 \%$ and specificity of $89.9 \%$ for the detection of DST (including the superior sagittal, sigmoid, and transverse sinuses). ${ }^{12}$

T1, T2, and FLAIR had false-negative rates up to $40 \%$, thought to be likely secondary to previously described signal characteristics of acute thrombus, which can mimic normal flow voids, in the dural sinuses, reviewed by Bonneville ${ }^{24}$ in 2014, and have been described as a factor underlying the false-negative results in noncontrast MRV-DST detection. We observed that GRE T2* blooming artifacts alone detected only 8/16 (50\%) cases of TST + . This is also presumed to be due to changes in $\mathrm{T}^{*}$ signal during the evolution from the acute-to-subacute phase, ${ }^{24}$ supporting $\mathrm{T} 2 *$ blooming artifacts being sensitive for acute thrombus but less so for subacute-to-chronic thrombi (as characterized by T1, T2, and FLAIR hyperintense TS signal). However, at least 2 prior studies have reported that GRE is highly sensitive to not only acute but also subacute thrombosis (also characterized by T1, T2, and FLAIR hyperintense signal), though not for chronic DST. $^{25,26}$ Of note, Altinkaya et $\mathrm{al}^{26}$ has demonstrated that the sensitivity of GRE for TST + and sigmoid sinus thrombosis is decreased due to artifacts from the skull base, potentially explaining the discrepancy between the prior studies and the current study, which specifically focused on the TS.

Fifty-six of 192 (29.2\%) PC-MRVs without TST+ demonstrated NVTS. Of these, the left TS was NV $23.4 \%$ of the time and was considered smaller versus the right TS in $24.0 \%$ of cases. The right TS was NV in $5.7 \%$ of cases and was considered smaller versus the left TS in $3.1 \%$ of cases. These findings are concordant with reports across multiple imaging modalities showing that the left and right TSs are hypoplastic in 39\%-60\% and 6\%-14\% of patients, respectively. ${ }^{27-29}$ The percentages reported in the current work are lower than of those previously reported, likely secondary to our strict criteria of $\mathrm{NV}$ as $>50 \%$ nonvisualized relative to the contralateral side.

Our limited dataset demonstrates no association with hypoplastic TS and TST + , in disagreement with a recent case-control report correlating TS hypoplasia and increased relative risk for TST $+{ }^{28}$ However, an earlier study described the opposite finding that the dominant TS was more likely to thrombose, suggesting that more work will be needed in the future to elucidate whether there is a correlation between TST + and TS size. ${ }^{30}$

This study has several limitations. First, the study inclusion criteria of patients who had PC-MRV, noncontrast MR imaging, and MPRAGE + performed within 24 hours of each other meant that not all patients with TST + were selected. Thus, the true 
prevalence of the disease, positive predictive value, negative predictive value, and accuracy could not be calculated, potentially decreasing the generalizability of the results and introducing unforeseen selection bias. Second, because the study was designed specifically to assess the utility of standard MR imaging sequences in the diagnosis of TS thrombosis in a subset of patients with presumed higher pretest probability based on abnormal noncontrast MRV findings, we did not perform an analysis of patients with normal MRV imaging findings. While it is known that conventional MR imaging sequences result in false-positive detection for DST (specificity of $14 \%-89.9 \%$ ), a comparison of conventional MR imaging sequence performance in patients without NVTS for detection of TS thrombosis in the current study with prior work could have yielded information on population differences and selection biases. ${ }^{12,22}$

Third, although MPRAGE + is superior to PC-MRV and MRI- in the diagnosis of TST and is thus established as the diagnostic criterion standard for TST+ in this study, some authors have shown that MPRAGE+ demonstrates decreased sensitivity compared with CE-MRV., ${ }^{9,0}$ This lowered sensitivity has been theorized to be from the time delay following gadolinium administration to image acquisition, causing enhancement of subacute thrombus, thus resulting in false-negative findings. This outcome could result in underreporting of true TST + in our study and decreases the sensitivity and specificity of noncontrast MR imaging sequences for thrombus detection. Future work using CE-MRV as the diagnostic criterion standard may be required. Fourth, because the diagnosis of DST of the superior sagittal sinus, internal cerebral veins, vein of Galen, and straight sinus is typically less of a diagnostic challenge on noncontrast MRV due to significantly fewer anatomic variations versus the TS, this study did not assess venous anatomy or MRI- signal on the remainder of the dural venous sinuses, limiting the generalizability of the results to DST other than TST + . Finally, our selection criteria excluded patients with known chronic TST + , and our current findings cannot necessarily be extended to the detection of chronic thrombosis. Future work is needed to assess the clinical utility of DWI for thrombi in the more chronic phase.

\section{CONCLUSIONS}

Unilateral NVTS on noncontrast MRV is a frequent phenomenon and presents a diagnostic challenge in determining whether the findings represent a TS anatomic variation, downstream venous effects, or technical artifacts versus true thrombosis. In patients in whom gadolinium contrast is contraindicated or refused, the DWI sequence can be effectively used to help exclude TST + in the setting of an equivocal noncontrast MRV.

\section{REFERENCES}

1. Stam J. Thrombosis of the cerebral veins and sinuses. $N$ Engl J Med 2005;352:1791-98 CrossRef Medline

2. Coutinho JM, Zuurbier SM, Aramideh M, et al. The incidence of cerebral venous thrombosis: a cross-sectional study. Stroke 2012;43: 3375-77 CrossRef Medline

3. Konakondla S, Schirmer CM, Li F, et al. New developments in the pathophysiology, workup, and diagnosis of dural venous sinus thrombosis (DVST) and a systematic review of endovascular treatments. Aging Dis 2017;8:136-48 CrossRef Medline

4. Coutinho JM. Cerebral venous thrombosis. J Thromb Haemost 2015;13(Suppl 1):S238-44 CrossRef Medline

5. Coutinho JM, Zuurbier SM, Stam J. Declining mortality in cerebral venous thrombosis: a systematic review. Stroke 2014;45:1338-41 CrossRef Medline

6. Miranda B, Ferro JM, Canhão P, et al. Venous thromboembolic events after cerebral vein thrombosis. Stroke 2010;41:1901-06 CrossRef Medline

7. Dmytriw AA, Song JS, Yu E, et al. Cerebral venous thrombosis: state of the art diagnosis and management. Neuroradiology 2018;60:66985 CrossRef Medline

8. Khandelwal N, Agarwal A, Kochhar R, et al. Comparison of CT venography with MR venography in cerebral sinovenous thrombosis. AJR Am J Roentgenol 2006;187:1637-43 CrossRef Medline

9. Saindane AM, Mitchell BC, Kang J, et al. Performance of spin-echo and gradient-echo T1-weighted sequences for evaluation of dural venous sinus thrombosis and stenosis. AJR Am J Roentgenol 2013;201:162-69 CrossRef Medline

10. Sari S, Verim S, Hamcan S, et al. MRI diagnosis of dural sinus: cortical venous thrombosis: Immediate post-contrast 3D GRE T1-weighted imaging versus unenhanced MR venography and conventional MR sequences. Clin Neurol Neurosurg 2015;134:44-54 CrossRef Medline

11. Liang L, Korogi Y, Sugahara T, et al. Evaluation of the intracranial dural sinuses with a 3D contrast-enhanced MP-RAGE sequence: prospective comparison with $2 \mathrm{D}$-TOF MR venography and digital subtraction angiography. AJNR Am J Neuroradiol 2001;22:481-92 Medline

12. Patel D, Machnowska M, Symons S, et al. Diagnostic performance of routine brain MRI sequences for dural venous sinus thrombosis. AJNR Am J Neuroradiol 2016;37:2026-32 CrossRef Medline

13. McCollough $\mathrm{CH}$, Schueler BA, Atwell TD, et al. Radiation exposure and pregnancy: when should we be concerned? Radiographics 2007;27:909-17; discussion 917-18 CrossRef Medline

14. Guo BJ, Yang ZL, Zhang LJ. Gadolinium deposition in brain: current scientific evidence and future perspectives. Front Mol Neurosci 2018;11:335 CrossRef Medline

15. Ayanzen RH, Bird CR, Keller PJ, et al. Cerebral MR venography: normal anatomy and potential diagnostic pitfalls. AJNR Am J Neuroradiol 2000;21:74-78 Medline

16. Han K, Chao AC, Chang FC, et al. Diagnosis of transverse sinus hypoplasia in magnetic resonance venography: new insights based on magnetic resonance imaging in combined dataset of venous outflow impairment case-control studies: post hoc case-control study. Medicine (Baltimore) 2016;95:e2862 CrossRef Medline

17. Chik Y, Gottesman RF, Zeiler SR, et al. Differentiation of transverse sinus thrombosis from congenitally atretic cerebral transverse sinus with CT. Stroke 2012;43:1968-70 CrossRef Medline

18. Provenzale JM, Kranz PG. Dural sinus thrombosis: sources of error in image interpretation. AJR Am J Roentgenol 2011;196:23-31 CrossRef Medline

19. Morris PP, Black DF, Port J, et al. Transverse sinus stenosis is the most sensitive MR imaging correlate of idiopathic intracranial hypertension. AJNR Am J Neuroradiol 2017;38:471-77 CrossRef Medline

20. Leach JL, Jones BV, Tomsick TA, et al. Normal appearance of arachnoid granulations on contrast-enhanced CT and MR of the brain: differentiation from dural sinus disease. AJNR Am J Neuroradiol 1996;17:1523-32 [Mismatch]Medline

21. Landis JR, Koch GG. The measurement of observer agreement for categorical data. Biometrics 1977;33:159-74 CrossRef Medline

22. Sadigh G, Mullins ME, Saindane AM. Diagnostic performance of MRI sequences for evaluation of dural venous sinus thrombosis. AJR Am J Roentgenol 2016;206:1298-306 CrossRef Medline

23. Yildiz ME, Ozcan UA, Turk A, et al. Diffusion-weighted MR imaging findings of cortical vein thrombosis at 3 T. Clin Neuroradiol 2015;25:249-56 CrossRef Medline

24. Bonneville F. Imaging of cerebral venous thrombosis. Diagn Interv Imaging 2014;95:1145-50 CrossRef Medline 
25. Ihn YK, Jung WS, Hwang SS. The value of T2*-weighted gradientecho MRI for the diagnosis of cerebral venous sinus thrombosis. Clin Imaging 2013;37:446-50 CrossRef Medline

26. Altinkaya N, Demir S, Alkan O, et al. Diagnostic value of T2*-weighted gradient-echo MRI for segmental evaluation in cerebral venous sinus thrombosis. Clin Imaging 2015;39:15-19 CrossRef Medline

27. Manara R, Mardari R, Ermani M, et al. Transverse dural sinuses: incidence of anatomic variants and flow artifacts with $2 \mathrm{D}$ time-offlight MR venography at 1 Tesla. Radiol Med 2010;115:326-38 CrossRef Medline
28. Arauz A, Chavarria-Medina M, Patiño-Rodriguez HM, et al. Association between transverse sinus hypoplasia and cerebral venous thrombosis: a case-control study. J Stroke Cerebrovasc Dis 2018;27:432-37 CrossRef Medline

29. Matsuda W, Sonomura T, Honma S, et al. Anatomical variations of the torcular Herophili: macroscopic study and clinical aspects. Anat Sci Int 2018;93:464-68 CrossRef Medline

30. Glik A, Benkovich E, Kesler A, et al. Lateral sinus thrombosis: the importance of the unaffected sinus. J Neuroimaging 2016;26:599604 CrossRef Medline 